\title{
Comment on: Sciatica in the Young
}

\author{
Farzad Omidi-Kashani ${ }^{1}$, Ebrahim Ghayem Hasankhani ${ }^{1}$, Ehsan Rafeemanesh ${ }^{2}$ \\ ${ }^{1}$ Orthopedic Department, Orthopedic Research Center, Imam Reza Hospital, Mashhad University of Medical Sciences, Mashhad, Iran \\ ${ }^{2}$ Occupational Medicine Department, Faculty of Medicine, Mashhad University of Medical Sciences, Mashhad, Iran
}

This letter is written in response to a note recently published regarding to the case report entitled "sciatica in a five year old boy" by Omidi-Kashani et al. [1] which had been published in June 2014 issue in the "Asian Spine Journal" [1]. We agree with the authors in that pediatric sciatica is commonly caused by lumbar disc herniation, although apophyseal ring fracture may even be more prevalent in this age group, despite the fact that both have very low incidences and are infrequently encountered in the literature $[2,3]$. Another important point worth mentioning regarding pediatric sciatica, is the lower incidences of secondary neurologic deficits due to increased resilience of the neural tissue in pediatric patients. This resilience often makes physical findings usually seen in adult patients such as muscular weakness, paresthesias, or impaired reflexes less pronounced thus limiting the exam where a positive straight leg raising may be the only positive finding [4]. Lastly, it is important that spondylolysis and spondylolisthesis are also kept in mind before considering the more unusual causes, especially in this young population [5].

\section{Conflict of Interest}

No potential conflict of interest relevant to this article was reported.

\section{References}

1. Omidi-Kashani F, Hasankhani EG, Rafeemanesh E. Sciatica in a five-year-old boy. Asian Spine J 2014;8:357-60.

2. Benifla M, Melamed I, Barrelly R, Aloushin A, Shelef I. Unilateral partial hemilaminectomy for disc removal in a 1 -year-old child. J Neurosurg Pediatr 2008;2:133-5.

3. Wu X, Ma W, Du H, Gurung K. A review of current treatment of lumbar posterior ring apophysis fracture with lumbar disc herniation. Eur Spine J 2013; 22:475-88

4. Bradbury N, Wilson LF, Mulholland RC. Adolescent disc protrusions: a long-term follow-up of surgery compared to chymopapain. Spine 1996; 21: 372-7.

5. Altaf F, Heran MK, Wilson LF. Back pain in children and adolescents. Bone Joint J 2014;96B:717-23.

Received Sep 10, 2014; Accepted Sep 10, 2014

Corresponding author: Farzad Omidi-Kashani

Orthopedic Research Center, Orthopedic Department, Imam Reza Hospital, Imam Reza Square, Mashhad University of Medical Sciences, Mashhad, Iran

Tel: +98-915-514-9248, Fax: +98-51-3859-5023, E-mail: omidif@mums.ac.ir 